MMCA-97 Conference, pp. 35-40

R. Čiegis (Ed)

(C) 1997 Vilniaus Gedimino technikos universitetas

\title{
SOLUTIONS OF A NONLINEAR DIRICHLET PROBLEM IN WHICH THE NONLINEAR PART IS BOUNDED FROM ABOVE AND BELOW BY POLYNOMIALS
}

JAN BȨCZEK

Institute of Mathematics and Physics, Pedagogical University, ul. Żotnierska 14, PL-10561 Olsztyn, Poland,

\section{ABSTRACT}

In this paper we study the existence and multiplicity solutions of nonlinear elliptic problem of the form

$$
\begin{aligned}
\Delta u+\lambda \cdot u-f(u) \cdot u & =g & & \text { in } \Omega \\
u & =0 & & \text { on } \delta \Omega
\end{aligned}
$$

Here, $\Omega$ is a smooth and bounded domain in $R^{N}, \quad N \geq 2, \lambda \in R$ and $f: R \longrightarrow R$ is a continuous, even function satisfying the following condition

$$
\begin{aligned}
c_{1} \cdot|t|^{\alpha} & \leq f(t) \leq c_{2} \cdot|t|^{p-1} & & \text { for }|t|>1 \\
0 & \leq f(t) \leq c_{3} \cdot|t| & & \text { for }|t| \leq 1
\end{aligned}
$$

for some $c_{1}, c_{2}, c_{3}, p, \alpha \in R, c_{1}, c_{2}, c_{3}, \alpha>0$ and $p>1+\alpha$.

We shall show that, for $\lambda \in R, g \in L_{r}(\Omega)$ if $N=2, r>1, p>1+\alpha$ or

$N \geq 3, r \geq \frac{2 \cdot N}{N+2}, \quad 1+\alpha<p<\frac{N+2}{N-2}$, the above problem has solutions.

Assuming additionally that, $\lambda \leq \lambda_{1}$ and $\mathrm{f}$ is decreasing for $t \leq 0$, we shall show that, this problem have exctly one solution.

We take advantage of the fact, that a continuous, proper and odd (injective) map of the form $\mathrm{I}+\mathrm{C}$ (where $\mathrm{C}$ is compact) is suriective (a homeomorphism).

\section{THE MAIN RESULTS}

We will consider the following nonlinear Dirichlet problem

$$
\begin{aligned}
\Delta u+\lambda \cdot u-f(u) \cdot u & =g & & \text { in } \Omega \\
u & =0 & & \text { on } \delta \Omega
\end{aligned}
$$

Here, $\Omega$ is a smooth and bounded domain in $R^{N}, \quad N \geq 2, \lambda \in R$ and $f: R \longrightarrow R$ is a continuous, even function satisfying the following condition 


$$
\begin{array}{ll}
c_{1} \cdot|t|^{\alpha} \leq f(t) \leq c_{2} \cdot|t|^{p-1} & \text { for }|t|>1 \\
0 \leq f(t) \leq c_{3} \cdot|t| & \text { for }|t| \leq 1
\end{array}
$$

for some $c_{1}, c_{2}, c_{3}, p, \alpha \in R, c_{1}, c_{2}, c_{3}, \alpha>0$ and $p>1+\alpha$.

We assume that, $p>1+\alpha$ for $N=2$ and $1+\alpha<p<\frac{N+2}{N-2}$ for $N \geq 3$.

For each $u \in \stackrel{\circ}{W}_{12}(\Omega)$, let us define the elements $L u, T u$ as follows:

$$
\begin{aligned}
& (L u, \phi)_{12}=\int_{\Omega} u \cdot \phi d x \\
& (T u, \phi)_{12}=\int_{\Omega} f(u) \cdot u \cdot \phi d x
\end{aligned}
$$

for every $\phi \in \stackrel{\circ}{W}_{12}(\Omega)$.

Lemma 1. If $u \in \stackrel{\circ}{W}_{12}(\Omega)$, then $L u, T u \in \stackrel{\circ}{W}_{12}(\Omega)$.

P r o o f. Let $u \in \stackrel{\circ}{W}_{12}(\Omega)$. Then, for every $\phi \in \stackrel{\circ}{W}_{12}(\Omega)$, we have

$$
\left|q_{1}(\phi)\right|=\left|\int_{\Omega} u \cdot \phi d x\right| \leq \int_{\Omega}|u \cdot \phi| d x \leq\|u\|_{02} \cdot\|\phi\|_{02} \leq c \cdot\|\phi\|_{12}
$$

and

$$
\begin{aligned}
\left|q_{2}(\phi)\right| & =\left|\int_{\Omega} f(u) \cdot u \cdot \phi d x\right| \leq \int_{\Omega}|f(u) \cdot u \cdot \phi| d x \\
& \leq\|f(u) \cdot u\|_{0 \frac{2 \cdot N}{N+2}} \cdot\|\phi\|_{0 \frac{2 \cdot N}{N+2}} \leq c \cdot\|\phi\|_{12}
\end{aligned}
$$

for $N \geq 3$ or

$$
\left|q_{2}(\phi)\right| \leq\|f(u) \cdot u\|_{02} \cdot\|\phi\|_{02} \leq c \cdot\|\phi\|_{12}
$$

when $\mathrm{N}=2$.

This means that, functions $q_{1}$ and $q_{2}$ are linear continuous functionals defined on the Hilbert space $\stackrel{\circ}{W}_{12}(\Omega)$. The Riesz theorem implies that, $L u, T u \in \stackrel{\circ}{W}_{12}(\Omega)$.

Thus we can define the operators $L, T: \stackrel{\circ}{W}_{12}(\Omega) \longrightarrow \stackrel{\circ}{W_{12}}(\Omega)$. Now we shall prove some properties of $\mathrm{L}$ and $\mathrm{T}$.

Lemma 2. The operator $L$ is linear and compact.

$\mathrm{P}$ r o o f. It is obvious that, $\mathrm{L}$ is linear. Let us consider a bounded sequence $\left\{u_{n}\right\}$ in $\stackrel{\circ}{W}_{12}(\Omega)$. Since the imbedding $\stackrel{\circ}{W}_{12}(\Omega) \subset L_{2}(\Omega)$ is compact, there exists a subsequence $\left\{u_{n_{k}}\right\}$ such that $u_{n_{k}} \longrightarrow u$ in $L_{2}(\Omega), k \longrightarrow \infty$. Hence 


$$
\left\|L u_{n_{k}}-L u\right\|_{12} \leq c \cdot\left\|u_{n_{k}}-u\right\|_{02} \longrightarrow 0
$$

This means that, $L u_{n_{k}} \longrightarrow L u$ in $W_{12}^{\circ}(\Omega)$ as $k \longrightarrow \infty$ and the operator L is compact. $\square$

Lemma 3. The map $T$ is continuous and compact.

P r o o f. Let $u_{n} \longrightarrow u$ in $\stackrel{\circ}{W}_{12}(\Omega), \quad n \longrightarrow \infty$. The continuity of the imbedding $\stackrel{\circ}{W_{12}}(\Omega) \subset L_{\frac{2 \cdot N}{N-2}}(\Omega)$ implies that $u_{n} \longrightarrow u$ in $L_{\frac{2 \cdot N}{N-2}}(\Omega)$.

It follows from inequality (2) and the Vajnberg theorem that, $f\left(u_{n}\right) \cdot u \longrightarrow$ $f(u) \cdot u$ in $L_{\frac{2 \cdot N}{(N-2) \cdot p}}(\Omega)$. Hence

$$
\begin{aligned}
\left\|T u_{n}-T u\right\|_{12} & =\sup _{\|\phi\|_{12}=1} \mid\left(T\left(u_{n}-T u, \phi\right)_{12} \mid\right. \\
& =\sup _{\|\phi\|_{12}=1} \mid \int_{\Omega}\left(f\left(u_{n}\right) \cdot u_{n}-f(u) \cdot u\right) \cdot \phi d x \\
& \leq \sup _{\|\phi\|_{12}=1}\left\|f\left(u_{n}\right) \cdot u_{n}-f(u) \cdot u\right\|_{0 \frac{2 \cdot N}{N+2}} \cdot\|\phi\|_{0 \frac{2 \cdot N}{N-2}} \\
& \leq c \cdot \sup _{\|\phi\|_{12}=1}\left\|f\left(u_{n}\right) \cdot u_{n}-f(u) \cdot u\right\|_{0 \frac{2 \cdot N}{N+2}} \cdot\|\phi\|_{12} \\
& \leq c \cdot\left\|f\left(u_{n}\right) \cdot u_{n}-f(u) \cdot u\right\|_{0 \frac{2 \cdot N}{N+2}} \longrightarrow 0
\end{aligned}
$$

for $n \longrightarrow \infty, N>2$ and

$$
\begin{aligned}
\left\|T u_{n}-T u\right\|_{12} & \leq \sup _{\|\phi\|_{12}=1}\left\|f\left(u_{n}\right) \cdot u_{n}-f(u) \cdot u\right\|_{02} \cdot\|\phi\|_{02} \\
& \leq c\left\|f\left(u_{n}\right) \cdot u_{n}-f(u) \cdot u\right\|_{02} \longrightarrow 0
\end{aligned}
$$

for $N=2$.

This proves continuity of $T$.

Now let $\left\{u_{n}\right\}$ be a bounded sequence in $\stackrel{\circ}{W}_{12}(\Omega)$. From the compactness of the imbedding $\stackrel{\circ}{W}_{12}(\Omega) \subset L_{\frac{2 \cdot N \cdot p}{N+2}}(\Omega)$, we conclude that, there exists a subsequence $\left\{u_{n_{k}}\right\}$ such that $u_{n_{k}} \longrightarrow$ win $L_{\frac{2 \cdot N \cdot p}{N+2}}(\Omega), k \longrightarrow \infty$. It follows from inequality (2) and the Vajnberg theorem that, $f\left(u_{n_{k}}\right) \cdot u_{n_{k}} \longrightarrow$ $f(w) \cdot w$ in $L_{\frac{2 \cdot N}{N+2}}(\Omega)$. The continuity of $\mathrm{T}$ implies that $\mathrm{T}$ is compact. $\square$

Let $A: \stackrel{\circ}{W}_{12}(\Omega) \longrightarrow \stackrel{\circ}{W_{12}}(\Omega)$ be a map defined by

$$
A=I-\lambda \cdot L+T .
$$

We shall prove some properties of A. 
Lemma 4. The map is odd.

P r o o f. $A(-u)=(-u)-\lambda \cdot L(-u)+T(-u)=-u+\lambda \cdot L u-T u=$ $-(u-\lambda \cdot L u+T u)=-A u$. $\square$

Lemma 5. The map $A$ is proper.

P r o o f. We shall show that, $\|A u\|_{12} \longrightarrow \infty$ if $\|u\|_{12} \longrightarrow \infty$. Let $\left\{u_{n}\right\} \subset \stackrel{\circ}{W 12}_{12}(\Omega)$ and $\left\|u_{n}\right\|_{12} \longrightarrow \infty$ as $n \longrightarrow \infty$. Then

$$
\begin{aligned}
\left(A u_{n}, u_{n}\right)_{12} & =\left(u_{n}, u_{n}\right)_{12}-\lambda \cdot\left(L u_{n}, u_{n}\right)_{12}+\left(T u_{n}, u_{n}\right)_{12} \\
& =\|u\|_{12}^{2}-\lambda \cdot\|u\|_{02}^{2}+\int_{\Omega} f\left(u_{n}\right) \cdot u_{n}^{2} d x \\
& \geq\left\|u_{n}\right\|_{12}^{2}-\lambda \cdot\left\|u_{n}\right\|_{02}^{2}+\int_{\Omega}\left|u_{n}\right|^{2+\alpha} d x \\
& =\left\|u_{n}\right\|_{12}^{2}-\lambda \cdot\left\|u_{n}\right\|_{02}^{2}+\left\|u_{n}\right\|_{02+\alpha}^{2+\alpha} \\
& \geq\left\|u_{n}\right\|_{12}^{2}-\lambda \cdot\left\|u_{n}\right\|_{02}^{2}+c \cdot\left\|u_{n}\right\|_{02}^{2+\alpha} \\
& =\left\|u_{n}\right\|_{12}^{2}+\left\|u_{n}\right\|_{02}^{2}\left(c \cdot\left\|u_{n}\right\|_{02}^{\alpha}-\lambda\right) .
\end{aligned}
$$

But $\left(A u_{n}, u_{n}\right)_{12} \leq\left\|A u_{n}\right\|_{12} \cdot\left\|u_{n}\right\|_{12}$, therefore

$\left\|A u_{n}\right\|_{12} \geq\left\|u_{n}\right\|_{12}+\left\|u_{n}\right\|_{02}^{2}\left(c \cdot\left\|u_{n}\right\|_{02}^{\alpha}-\lambda\right) /\left\|u_{n}\right\|_{12} \longrightarrow \infty$ as $n \longrightarrow \infty$.

TheOrem 1. The map $A$ is suriective.

$\mathrm{P}$ r o o f. It follows from fact that, a continuous, proper and odd map of the form $\mathrm{I}+\mathrm{C}$ (where $\mathrm{C}$ is a compact) is suriective. $\square$

Theorem 2. If $\lambda \leq \lambda_{1}$ and $f$ is decreasing for $t \leq 0$, then $A$ is a homeomorphism.

$\mathrm{P}$ r o o f. We shall prove that $\mathrm{A}$ is injective. Let us assume that, there exist $u, v \in \stackrel{\circ}{W}_{12}(\Omega)$, such that $A u=A v$. Then

$$
\begin{gathered}
(A u, u-v)_{12}=(A v, u-v)_{12} \\
(u-v, u)_{12}-\lambda \cdot(L u, u-v)_{12}+(T u, u-v)_{12}- \\
-(v, u-v)_{12}+\lambda \cdot(L v, u-v)_{12}-(T v, u-v)_{12}=0 \\
(u-v, u-v)_{12}-\lambda \cdot(L u-L v, u-v)_{12}+(T u-T v, u-v)_{12}= \\
\|u-v\|_{12}^{2}-\lambda \cdot\|u-v\|_{02}^{2}+\int_{\Omega}(f(u) \cdot u-f(v) \cdot v) \cdot(u-v) d x=0 .
\end{gathered}
$$

Hence 


$$
\|u-v\|_{12}^{2}-\lambda \cdot\|u-v\|_{02}^{2}=-\int_{\Omega}(f(u) \cdot u-f(v) \cdot v) \cdot(u-v) d x .
$$

Since $\lambda \leq \lambda_{1}$, the left-hand side of the equation is nonnegative, the righthand side is nonpositive, so $u=v$ and therefore $\mathrm{A}$ is injective. From theorem 1 we conclude that, $\mathrm{A}$ is homeomorphism. $\square$

An element $u \in \stackrel{\circ}{W}_{12}(\Omega)$ is called a weak solution of problem (1), if the following condition is satisfied

$$
\int_{\Omega} \nabla u \cdot \nabla \phi d x-\lambda \cdot \int_{\Omega} u \cdot \phi d x+\int_{\Omega} f(u) \cdot u \cdot \phi d x=-\int_{\Omega} g \cdot \phi d x
$$

for every $\phi \in \stackrel{\circ}{W}_{12}(\Omega)$.

Let $g \in W_{-12}(\Omega)$, then there exists $h \in \stackrel{\circ}{W}_{12}(\Omega)$ such that $-\int_{\Omega} g \cdot \phi d x=$ $(h, \phi)_{12}$ and (4) can be written in the form

$$
(u-\lambda \cdot L u+T u, \phi)_{12}=(h, \phi)_{12}
$$

for every $\phi \in \stackrel{\circ}{W_{12}}(\Omega)$.

This equation is equivalent to

$$
u-\lambda \cdot L u+T u=h
$$

or

$$
A u=h .
$$

Theorem 3. If $g \in L_{r}(\Omega)$ where, $r>1, N=2, p>1+\alpha$ or $N \geq 3$, $r>\frac{2 \cdot N}{N+2}, 1+\alpha<p<\frac{N+2}{N-2}$, then there exists a weak solution of problem (1). If additionally $\lambda \leq \lambda_{1}$ and function $f$ is decreasing for $t \leq 0$, the problem (1) has exactly one weak solution.

$\mathrm{P}$ r o o f. Let g satisfies the assumptions of theorem. Then

$$
\left|\int_{\Omega}(-g) \cdot \phi d x\right| \leq\|g\|_{02} \cdot\|\phi\|_{02} \leq c \cdot\|\phi\|_{12} \quad \text { in case } N=2
$$

or

$$
\left|\int_{\Omega}(-g) \cdot \phi d x\right| \leq\|g\|_{0 \frac{2 \cdot N}{N+2}} \cdot\|\phi\|_{0 \frac{2 \cdot N}{N-2}} \leq c \cdot\|\phi\|_{12} \quad \text { for } N \geq 3
$$


It denotes that $g \in W_{-12}(\Omega)$. The thesis of theorem follows from theorems 1 and $2 . \square$

\section{REFERENCES}

[1] A. Ambrosetti and G. Prodi. On the inversion of some differentiable mappings with singularities between Banach spaces, Ann. Math. Pure Appl., 93, 1972, P. 213-246.

[2] A. Bahri and B. Berestycki. A perturbation method in critical point theory and applications, Trans. Amer. Math. Soc., 267, 1981, P. 1-30.

[3] M. S. Berger. Nonlinearity and functional analysis, Academic Press, New York, 1977.

[4] M. S. Berger and E. Podolak. On the solutions of a nonlinear Dirichlet problems, Indiana Univ. Math. J., 24, 1975, P. 837-846.

[5] J. Bęczek. On solutions of some nonlinear Dirichlet problems, Zeszyty naukowe UG, 1987, P. 7-15.

[6] J. Bęczek. On the existence of solutions of some nonlinear Dirichlet problems, Annales Polonici Math., XLIX, 1988, P. 45-51.

[7] E. N. Dancer. On the Dirichlet Problem for Weakly Nonlinear Elliptic Partial Differential Equations, Proc. Royal. Soc., Edinburgh 76 A , 1977, P. 283-300.

[8] J. Dugundi and A. Granas. Fixed point theory, PWN Warszawa, 1982.

[9] O. Ladyzenskaya and N. Uralceva. Linear and Quasilinear Elliptic Partial Differential Equations, Academic Press, New York, 1968.

[10] L. Nirenberg. Topics in Nonlinear Functional Analysis, Courant Inst. of Math. Sciences, New York, 1974. 